\title{
KDM3A regulates Slug expression to promote the invasion of MCF7 breast cancer cells in hypoxia
}

\author{
HYUN-JUNG AHN ${ }^{1}$, BYUL MOON ${ }^{1,2}$, MIJIN PARK ${ }^{1,2}$ and JUNG-AE KIM ${ }^{1,2}$ \\ ${ }^{1}$ Personalized Genomic Medicine Research Centre, Korea Research Institute of Bioscience and Biotechnology, Daejeon 34141; \\ ${ }^{2}$ Department of Functional Genomics, Korea Research Institute of Bioscience and Biotechnology School of Bioscience, \\ University of Science and Technology, Daejeon 34113, Republic of Korea
}

Received June 19, 2020; Accepted September 18, 2020

DOI: $10.3892 / \mathrm{ol} .2020 .12199$

\begin{abstract}
Oxygen deprivation (hypoxia), which frequently occurs in the tumour microenvironment, is a strong driver of the phenotypic transition of cancer cells. An increase in metastatic potential such as cell invasion is a well-known phenotypical change induced in hypoxia. The present study demonstrated that lysine demethylase 3A (KDM3A), a Jumonji C domain-containing $\mathrm{KDM}$, is involved in the hypoxia-induced invasion of MCF7 breast cancer cells. KDM3A depletion inhibits the induction of cell invasion without affecting MCF7 cell survival rate or proliferation under hypoxic conditions, whereas KDM3A overexpression enhances MCF7 cell invasion even under normoxic conditions. KDM3A suppresses E-cadherin expression, which is associated with epithelial-to-mesenchymal transition (EMT)-mediated cell invasion in hypoxia. In addition, KDM3A promotes the expression of Slug, an EMT transcription factor that negatively regulates E-cadherin expression. Consistent with this finding, the removal of the repressive transcription marker, dimethylated histone $\mathrm{H} 3$ at lysine 9 from the Slug promoter is dependent on hypoxia-induced recruitment of KDM3A. Collectively, the results of the present study suggest that KDM3A is a crucial transcriptional coactivator of Slug expression to induce MCF7 breast cancer cell invasion in hypoxia, and that inhibition of KDM3A may efficaciously prevent metastatic cancer development.
\end{abstract}

\section{Introduction}

One of the most difficult challenges in developing anticancer treatment strategies is overcoming the phenotypic plasticity of cancer cells. Oxygen deprivation (also known as hypoxia) to a level lower than the physiological concentration is frequently

Correspondence to: Dr Jung-Ae Kim, Personalized Genomic Medicine Research Centre, Korea Research Institute of Bioscience and Biotechnology, 125 Gwahak-ro, Yuseong, Daejeon 34141, Republic of Korea

E-mail: jungaekim@kribb.re.kr

Key words: KDM3A, hypoxia, breast cancer, cancer cell invasion, Slug accompanied by the rapid proliferation of cancer cells in vivo, and functions as a strong extracellular factor to stimulate cancer cell plasticity (1). In hypoxia, cancer cells induce various response pathways. To provide the energy necessary for cellular activities when the oxygen supply is scarce, cells upregulate anaerobic glycolysis-associated pathways (2). Angiogenesis is also stimulated to generate blood vessels for tumour growth (3). Furthermore, hypoxia enables the induction of the epithelial-to-mesenchymal transition (EMT) and subsequent cell motility, which reflect the phenotypical transition of cancer cells to aggressive metastasis (1).

The reprogramming of gene expression and/or signalling pathways is involved in hypoxia-induced changes in the state of cancer cells. Hypoxia-inducible factor-1 (HIF-1) is a key transcription factor involved in hypoxia-induced transcriptional alteration (4). Accumulation of HIF-1 in hypoxia alters the expression of numerous target genes, which mediates phenotypical changes in cancer cells. Chromatin alteration is also closely associated with hypoxia-induced transcriptional reprogramming. Among various chromatin modifications, histone lysine demethylation as a response to hypoxia is of particular interest, since Jumonji C (JmjC) domain-containing lysine demethylases (KDMs) require oxygen as a substrate for catalysis. While a lack of oxygen attenuates the activity of KDMs, the expression of multiple KDMs, some of which are HIF-1 dependent, is upregulated in hypoxic cells (5). Increased cellular levels of KDMs may compensate attenuated KDM catalytic activity. In addition, the direct molecular function of KDMs in hypoxia-induced responses have been reported: KDMs alter the chromatin structure of numerous target genes (6). These findings suggest that the mechanisms by which KDMs are involved in hypoxia-induced responses are important to understand the progression of aggressive forms of cancer.

Among the KDMs upregulated directly by HIF-1, KDM4C, which demethylates histone $\mathrm{H} 3$ at lysine 9 (H3K9), functions as a coactivator of HIF-1 and mediates breast cancer progression (7). KDM3A, another H3K9 demethylase, also enhances HIF-1 transcriptional activity. In endothelial cells, KDM3A associates with and demethylates $\mathrm{H} 3 \mathrm{~K} 9$ at the SLC2A3 locus in a HIF-dependent manner (8). KDM3A cooperates with HIF-1 to upregulate the expression of androgen receptor target genes in prostate cancer cells (9). KDM3A accumulation is 
also required for the proliferation of different cancer cell lines under hypoxic conditions (10). Furthermore, gene expression profiling of mouse embryonic stem cells under hypoxia revealed that KDM3A knockout affected the expression of numerous hypoxia-responsive genes (11). These findings collectively suggest that KDM3A is an important chromatin regulator for hypoxia-induced transcriptional reprogramming to mediate aggressive cancer progression. Accordingly, to gain insight into developing a means to overcome cancer cell plasticity in hypoxic tumour microenvironments, the present study investigated the molecular mechanisms by which KDM3A functions in cancer cell phenotypic transitions in hypoxia.

\section{Materials and methods}

Cell culture. The MCF7 cell line was purchased from the American Type Culture Collection and was maintained in high-glucose Dulbecco's modified Eagle's medium (DMEM; Gibco; Thermo Fisher Scientific, Inc.) supplemented with $10 \%$ foetal bovine serum (Gibco; Thermo Fisher Scientific, Inc.) and $1 \mathrm{X}$ antibiotic-antimycotic (Gibco; Thermo Fisher Scientific, Inc.). To provide hypoxic culture conditions where oxygen levels were lower than physiological normoxic levels $\left(21 \% \mathrm{O}_{2}\right)$, cells were cultured in $1 \%$ (for cell death, invasion, reporter assay and ChIP assay) or $5 \% \mathrm{O}_{2}$ (for cell proliferation assay) with $5 \% \mathrm{CO}_{2}$ (MCO-5M; Sanyo Electric Co., Ltd.), while normoxic culture conditions were maintained under $21 \% \mathrm{O}_{2}$ with $5 \% \mathrm{CO}_{2}$ (Heraeus $\mathrm{BB} 15 \mathrm{CO}_{2}$ Incubator; Thermo Fisher Scientific, Inc.).

Reagents, transfection and antibodies. Lipofectamine ${ }^{\circledR}$ Plus (Invitrogen; Thermo Fisher Scientific, Inc.) and RNAiMAX (Invitrogen; Thermo Fisher Scientific, Inc.) reagents were employed for DNA plasmid and small interfering (si)RNA transfection, respectively. siRNA against KDM3A (si-KDM3A; 5'-GUCUAUGUGGGAAUUCCCA-3') and a negative control (si-CTL; 5'-CCUACGCCACCAAUUUCGU-3') were purchased from Bioneer Corporation. The primary and secondary antibodies used in the present study were as follows: antiKDM3A (Santa Cruz Biotechnology, Inc.; sc-376608; 1:1,000), anti-E-cadherin (Santa Cruz Biotechnology, Inc.; sc-21791; 1:1,000), anti-Slug (Santa Cruz Biotechnology, Inc.; sc-1666477; 1:1,000), $\alpha$-Snail (Santa Cruz Biotechnology, Inc.; sc-271977; 1:1,000), anti-Twist (Santa Cruz Biotechnology, Inc.; sc-81417; 1:1,000), anti- $\beta$-actin (Santa Cruz Biotechnology, Inc.; sc-47778; 1:2,000), anti-Flag (Sigma-Aldrich; Merck KGaA; F3165; 1:1,000), anti-IgG chromatin immunoprecipitation (ChIP) grade (Abcam; ab2410; $1 \mu \mathrm{g} / \mathrm{ml}$ ) and horseradish peroxidase-conjugated anti-rabbit IgG (EMD Millipore; AP132P; 1:5,000). A plasmid construct encoding Flag-KDM3A derived from pcDNA3.1 was provided by Dr Hyun-Soo Cho (Korea Research Institute of Bioscience and Biotechnology). SNAI2 promoter activity reporter constructs were constructed by subcloning DNA fragments encompassing the transcriptional start site (TSS) of the SNAI2 gene into the Dual-Luciferase Reporter Assay System (Promega Corporation). The primers used for subcloning were as follows: Reporter (Rep)-A-forward (F), 5'-AAGGATACCGTTCCAAATGACAGTTAC-3'; Rep-B-F, 5'-AAGGATACCCGCCTTTGTCTTCCCGCTTCC-3'; and Rep-R, 5'-TTCTCGAGTTTTTGGAGGCGTTGAAATG-3'.
To generate different 5 ' regions of reporter constructs with the shared 3' region, a common Rep-R primer was used for the subcloning of both Rep-A and Rep-B constructs.

Cell proliferation assay. Proliferation of cells seeded at the same number $\left(1 \times 10^{5} /\right.$ well) in 6 -well plates under normoxic or hypoxic conditions was examined by counting the cell numbers 0,2 and 4 days after seeding. The number of viable cells with an automated cell counter (Invitrogen ${ }^{\mathrm{TM}}$ Countess $^{\mathrm{TM}}$ II FL Automated Cell Counter; Thermo Fisher Scientific, Inc.).

Apoptotic cell death measurement. Apoptotic cell death was measured using an Apo-ONE ${ }^{\text {TM }}$ Homogeneous Caspase-3/7 Assay kit (Promega Corporation). Assays were performed according to the manufacturer's protocols. Cells transfected with $0.1 \mu \mathrm{M}$ si-CTL or si-KDM3A were cultured in normoxic conditions $\left(21 \% \mathrm{O}_{2}\right)$ for $48 \mathrm{~h}$ and seeded in 96-well plates $\left(5 \times 10^{3}\right.$ cells/well), followed by incubation in normoxic $\left(21 \% \mathrm{O}_{2}\right)$ or hypoxic conditions $\left(1 \% \mathrm{O}_{2}\right)$ for $24 \mathrm{~h}$. The fluorescence signal (485-nm excitation wavelength/527-nm emission wavelength) generated from cleaved proluminescent caspase-3/7 DEVD-aminoluciferin substrate by active caspase-3/7 in apoptotic cells was recorded with a fluorometer (BioTek Instruments, Inc.).

Reverse transcription (RT)-PCR. Total RNA was isolated using TRIzol $^{\circledR}$ reagent (Invitrogen; Thermo Fisher Scientific, Inc.) and assessed with an ND-1000 spectrophotometer (NanoDrop Technologies; Thermo Fisher Scientific, Inc.). First-strand cDNA synthesis was produced with RevertAid ${ }^{\mathrm{TM}}$ M-MuLV Reverse Transcriptase (Thermo Fisher Scientific, Inc.) according to the manufacturer's protocols. RT-PCR was performed with AccuPower PCR Master Mix (Bioneer Corporation) and a GeneAmp PCR System 970 (Thermo Fisher Scientific, Inc.). The thermocycling conditions were as follows: Pre-denaturation at $94^{\circ} \mathrm{C}$ for $5 \mathrm{~min}$, followed by $20-25$ cycles of denaturation at $94^{\circ} \mathrm{C}$ for $20 \mathrm{sec}$, annealing at $55-60^{\circ} \mathrm{C}$ for $20 \mathrm{sec}$ and extension at $72^{\circ} \mathrm{C}$ for $20 \mathrm{sec}$, with a final extension at $72^{\circ} \mathrm{C}$ for $5 \mathrm{~min}$. The PCR products were electrophoresed on 1\%-agarose gels and stained with RedSafe ${ }^{\mathrm{TM}}$ (Intron, Inc.). PCR products were visualized with GelDoc ${ }^{\mathrm{TM}} \mathrm{XR}+$ (Bio-Rad Laboratories, Inc.) and ImageLab ${ }^{\text {TM }}$ v6.0.1 software (Bio-Rad Laboratories, Inc.) and semi-quantified by measuring densitometry using ImageJ (National Institutes of Health). The primers used for RT-PCR were as follows: KDM3A-F, 5'-CCAGCCTCAAAGGAAGACCT-3'; KDM3A-R, 5'-ACTGCACCAAGAGTCGGTTT-3'; E-cadherin (CDH1)-F, 5'-GCTTTGACGCCGAGAGCTACACGTT-3'; CDH1-R, 5'-AGGAGTTGGGAAATGTGAGCAATTC-3'; $\beta$-actin (ACTB)-F, 5'-GGAGTCCTGTGGCATCCACG-3'; and ACTB-R, 5'-CTAGAAGCATTTGCGGTGGA-3'.

Luciferase assay. Luciferase assay was performed using a Dual-Luciferase Reporter Assay System (Promega Corporation) according to the manufacturer's instructions. Cells $\left(7.5 \times 10^{4} /\right.$ well) cultured in 12 -well plates for $24 \mathrm{~h}$ were co-transfected with luciferase reporter $(0.2 \mu \mathrm{g})$, Renilla reporter $(0.05 \mu \mathrm{g})$ and si-CTL or KDM3A (100 nM). At $48 \mathrm{~h}$ post-transfection, the cells were exposed to hypoxic conditions for an additional $24 \mathrm{~h}$. Luciferase activities were determined using a luminometer (Luminoskan Ascent; Thermo Fisher 
Scientific, Inc.). Data represent the signals normalized to Renilla luciferase activity.

Immunoblot analysis. Cells were lysed in RIPA buffer (50 mM Tris- $\mathrm{HCl} \mathrm{pH} 7.5,150 \mathrm{mM} \mathrm{NaCl}, 10 \mathrm{mM}$ EDTA and $1 \%$ Triton X-100) with protease inhibitor cocktail (Roche Diagnostics) or $2 \mathrm{X}$ sample buffer $(120 \mathrm{mM}$ Tris- $\mathrm{HCl} \mathrm{pH} 6.8$, $20 \%$ glycerol, $4 \%$ SDS and 5\% $\beta$-mercaptoethanol). Proteins extracted with RIPA were quantified with the Bradford assay and equal amounts (30 $\mu \mathrm{g})$ of proteins were used for each sample. For resolving by SDS-PAGE, 5X SDS sample buffer [250 mM Tris-HCl (pH 6.8), 10\% SDS, 50\% glycerol, $0.5 \mathrm{M}$ dithiothreitol, $0.25 \%$ bromophenol] was added to cell lysates and boiled at $100^{\circ} \mathrm{C}$ for $10 \mathrm{~min}$. After SDS-PAGE, the proteins were transferred to nitrocellulose membranes and the membranes were blocked at room temperature for $1 \mathrm{~h}$ using $5 \%$ skimmed milk in TBST $(0.1 \%$ Tween- 20$)$ buffer. Primary antibodies were added and incubated at $4{ }^{\circ} \mathrm{C}$ for $16 \mathrm{~h}$, and then HRP-conjugated secondary antibody, which was incubated with the membranes at room temperature for $1 \mathrm{~h}$, was used for detecting the chemiluminescent signal (Pierce ${ }^{\mathrm{TM}} \mathrm{ECL}$ Western Blotting Substrate; Thermo Fisher Scientific, Inc.) from specific target proteins. The immunoblots were developed with LAS-4000 (Luminescent Image Analyzer; Fujifilm Wako Pure Chemical Corporation).

Invasion assays. The outsides of Transwell inserts were pre-coated with human collagen-type IV at room temperature for $1 \mathrm{~h}$. For the invasion assays, the upper chambers of the Transwell inserts were coated with $250 \mu \mathrm{g} / \mathrm{ml}$ Matrigel (Becton-Dickinson and Company) at room temperature for $1 \mathrm{~h}$. MCF7 cells were transfected with $0.1 \mu \mathrm{M}$ si-CTL or si-KDM3A. At $24 \mathrm{~h}$ post-transfection, the cells were incubated under either hypoxic $\left(1 \% \mathrm{O}_{2}\right)$ or normoxic $\left(21 \% \mathrm{O}_{2}\right)$ conditions After $24 \mathrm{~h}$ of incubation at different oxygen concentrations, the cells were harvested with $0.025 \%$ trypsin-EDTA and transferred to Transwell inserts $\left(1 \times 10^{5}\right.$ cells/chamber $)$ in 6 -well plates. After $24 \mathrm{~h}$ of incubation in normoxic conditions $\left(21 \% \mathrm{O}_{2}\right)$, the inserts were fixed with $100 \%$ methanol and stained with $0.5 \%$ crystal violet. The invaded cells were counted manually under a light microscope (x200 magnifications; 5 fields per replicate).

ChIP assay. ChIP assay was performed using a ChIP assay kit following the manufacturer's protocol (Upstate Biotechnology, Inc). Cells $\left(2 \times 10^{6}\right)$ were preincubated with a dimethyl 3,3'-dithiobispropionimidate- $\mathrm{HCl}$ solution $(5 \mathrm{mM}$; Pierce; Thermo Fisher Scientific, Inc.) for $30 \mathrm{~min}$ on ice and then treated with $1 \%$ formaldehyde at room temperature for $15 \mathrm{~min}$. ChIP-enriched DNA samples were visualized by PCR and electrophoresed on a $1.5 \%$-agarose gel stained with RedSafe ${ }^{\mathrm{TM}}$ (Intron, Inc.). Input DNA (1\%) used for ChIP with individual antibody served as the control. The thermocycling conditions were as follows: Pre-denaturation at $94^{\circ} \mathrm{C}$ for $5 \mathrm{~min}$, followed by 20 cycles of denaturation at $94^{\circ} \mathrm{C}$ for $20 \mathrm{sec}$, annealing at $55-60^{\circ} \mathrm{C}$ for $20 \mathrm{sec}$ and extension at $72^{\circ} \mathrm{C}$ for $15 \mathrm{sec}$, with a final extension at $72^{\circ} \mathrm{C}$ for $5 \mathrm{~min}$. Densitometry of visualized DNA was semi-quantitatively measured by ImageJ (National Institutes of Health). The primer pairs used for the ChIP assays, which were specific to regions within the SNAI2 promoter and the downstream adjacent DNA element (from $-1,500$ to $+1,000$ ) separated by a 200-bp interval, as labelled in Fig. 4A, were as follows: A-F, 5'-ACCTGACAATGCATTTTCTCT-3'; A-R, 5'-TCACATGAA GATCACCCTACTCT-3'; d-F, 5'-GGGTTCTTAAAATTTAA TCAATCTA-3'; d-R, 5'-AAAGCCATTAAAATCCATC TCA-3'; e-F, 5'-GTGAGAGAATGTCCGGTCCT-3'; e-R, 5'-GGCTGATCGGAAGAACTGGAA-3'; f-F, 5'-GCGGAA GCCCTGAGTAGC-3'; f-R, 5'-TTGAAAAAGGAAGGGGG AAGCGGGAAG-3'; B-F，5'-CCTTCTCCTGGCGGAC ACT-3'; B-R, 5'-ACGCTCTCTGGGAGCTAGG-3'; g-F, 5'-CTG TTCACAGCTGTCCCAGAG-3'; g-R, 5'-TTACGAACTGA GCCCGTTTT-3'; h-F, 5'-AGACCCGCTGGCAAGATG-3'; h-R, 5'-TCTTTTTACCTGTATGTGTCCA-3'; i-F, 5'-TTCGTT TGCTAAAGCCTGT-3'; i-R, 5'-GCCGCCTTCTAAAGGA-3'; C-F, 5'- AGCCCATACGTAACATGGAAAA-3'; C-R, 5'-TGC ACGGAGCTATAGGTGCTC-3'; j-F, 5'-CCATGCCTGTCA TACCACA-3'; and j-R, 5'-GAGAGGCCATTGGGTAGCTG-3'.

Statistical analysis. All data are presented as means of three independent experiments \pm standard deviation. GraphPad Prism 7 software (GraphPad Software, Inc.) was used for statistical analyses. Student's t-tests (two-tailed, unpaired) were used to determine significant differences between si-CTL versus si-KDM3A and Mock versus Flag-KDM3A in Fig. 1. In Fig. 1, data from normoxia $\left(21 \% \mathrm{O}_{2}\right)$ or hypoxia $\left(1 \% \mathrm{O}_{2}\right)$ groups were compared independently. Differences in KDM3A ChIP signals between normoxia $\left(21 \% \mathrm{O}_{2}\right)$ and hypoxia $\left(1 \% \mathrm{O}_{2}\right)$ in Fig. 4C was also analysed with Student's t-test (two-tailed, unpaired). Data in Figs. 3B, C and 4E were analysed with one-way ANOVA followed by Tukey's post hoc test to determine significant differences. $\mathrm{P}<0.05$ was considered to indicate a statistically significant difference.

\section{Results}

KDM3A is involved in breast cancer cell invasion in hypoxia. To examine the role of KDM3A in aggressive cancer cell behaviour in the hypoxic microenvironment, the involvement of KDM3A in hypoxia-induced cancer cell phenotypes was determined in the present study. Given the prominent clinical association between hypoxia-induced response and prognosis of patients with breast cancer (12), the function of KDM3A in MCF7 breast cancer cells was examined. First, the requirement of KDM3A for cell proliferation in hypoxia conditions was observed. It was notable that MCF7 cells incubated in $1 \% \mathrm{O}_{2}$ for longer than $24 \mathrm{~h}$ showed a higher death rate. Thus, cellular proliferation was determined in milder hypoxic conditions, $5 \% \mathrm{O}_{2}$, rather than $1 \% \mathrm{O}_{2}$. Under hypoxic conditions $\left(5 \% \mathrm{O}_{2}\right), \mathrm{MCF} 7$ cells proliferated at a $\sim 5$-fold decreased rate compared with that of MCF7 cells cultured in normoxic conditions $\left(21 \% \mathrm{O}_{2}\right.$; Fig. 1A). KDM3A depletion did not further aggravate MCF7 cell proliferation in hypoxic conditions. Notably, KDM3A knockdown minimally affected MCF7 cell proliferation in normoxic conditions. In addition, the loss of KDM3A did not trigger cell death in normoxic or hypoxic conditions $\left(1 \% \mathrm{O}_{2}\right)$, which was determined by the fluorescent signals generated from cleaved substrate by active caspases in apoptotic cells (Fig. 1B). These results indicate that KDM3A is dispensable for MCF7 cell survival and proliferation under hypoxic stress conditions. 
A

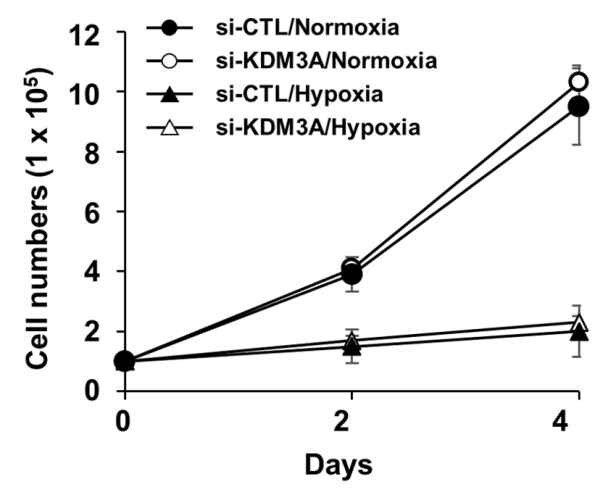

C

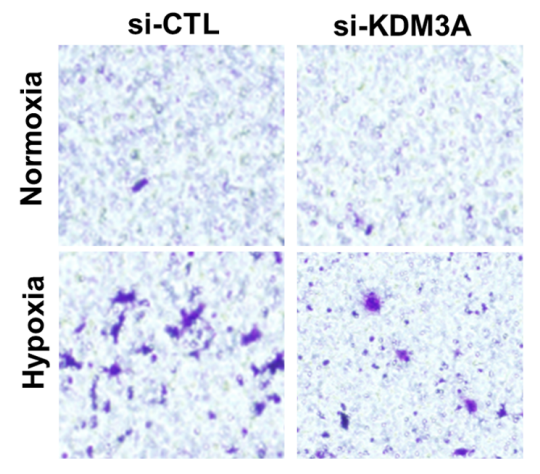

E

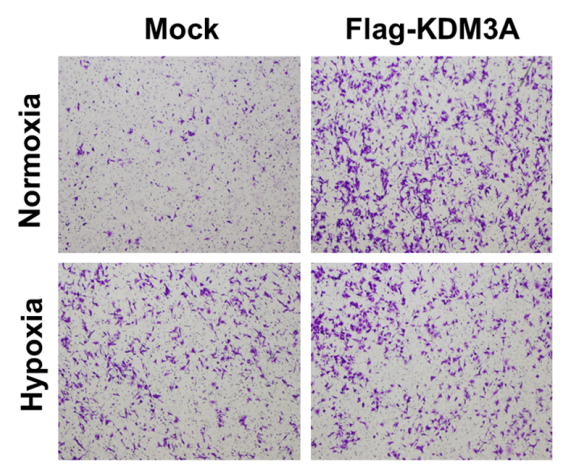

B

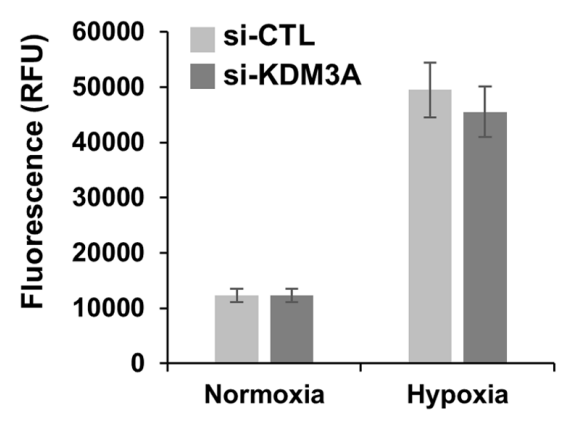

D

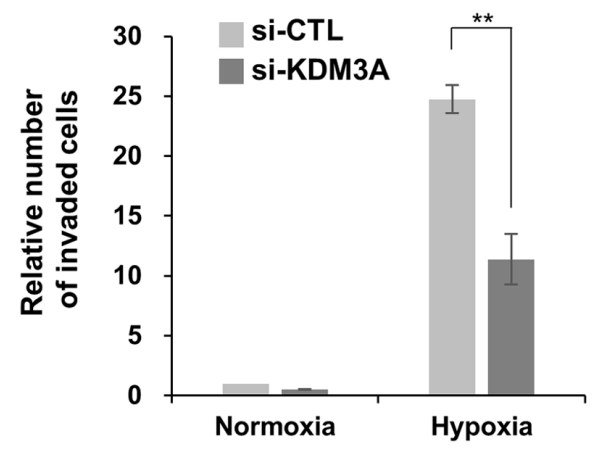

$\mathbf{F}$

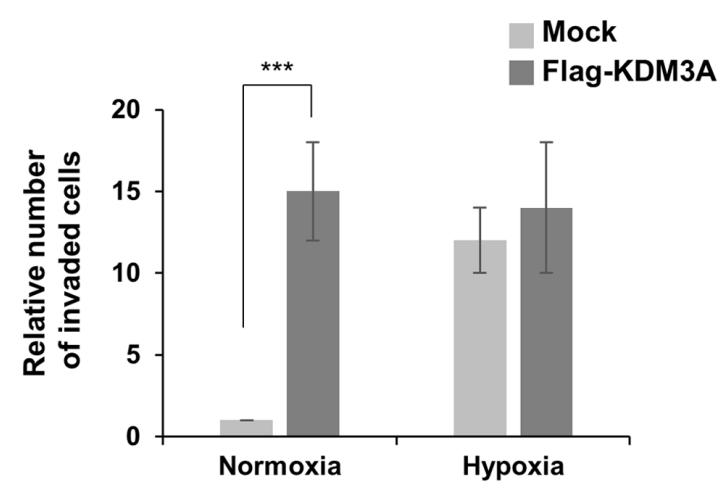

Figure 1. KDM3A is involved in hypoxia-induced breast cancer cell invasion. (A) KDM3A-depleted MCF7 cells had no effect on proliferation rate under normoxic $\left(21 \% \mathrm{O}_{2}\right)$ or hypoxic conditions $\left(5 \% \mathrm{O}_{2}\right)$. The graph shows the mean of three experiments. (B) KDM3A knockdown minimally affected the apoptosis-mediated death of MCF7 cells under normoxic $\left(21 \% \mathrm{O}_{2}\right)$ or hypoxic $\left(1 \% \mathrm{O}_{2}\right)$ conditions. Apoptotic cells were detected by measuring the fluorescent product generated by active caspase-3/7. The graph displays the mean of three experiments. (C and D) KDM3A depletion decreased the hypoxia-induced invasion of MCF7 cells. A representative image of three experiments showing crystal violet staining is shown in panel C. Magnification, $\mathrm{x} 200$. The quantification of invaded cells cultured under different conditions is shown in panel D. The number of invaded cells was normalized to that of si-CTL-transfected cells cultured under normoxic conditions. P-values were calculated with Student's t-test. (E and F) KDM3A overexpression mediated the invasion of MCF7 cells under normoxic conditions. Cells transfected with either empty vector (Mock) or Flag-tagged KDM3A (Flag-KDM3A)-encoded vector were cultured in normoxia for $24 \mathrm{~h}$, followed by culture under normoxic $\left(21 \% \mathrm{O}_{2}\right)$ or hypoxic conditions $\left(1 \% \mathrm{O}_{2}\right)$ for an additional $24 \mathrm{~h}$. Next, the cells were subjected to an invasion assay. A representative image of three experiments showing crystal violet staining is shown in panel E. Magnification, $\mathrm{x} 200$. The quantitation of the invaded cells is shown in panel F. The number of invaded cells was normalized to that of Mock-transfected cells cultured under nomoxic conditions. P-values were calculated using the Student's t-test. ${ }^{* *} \mathrm{P}<0.01$ and ${ }^{* * *} \mathrm{P}<0.001$. si, small interfering; CTL, control; KDM, lysine demethylase.

Next, the present study investigated whether KDM3A is associated with hypoxia-induced cell motility, another hypoxia-responsive cell phenotype that promotes the spread of aggressive cancer cells. MCF7 cells poorly invaded through Matrigel-coated Transwell chambers in normoxic conditions, whereas preincubation in hypoxia $\left(1 \% \mathrm{O}_{2}\right)$ for $24 \mathrm{~h}$ induced cell invasion (Fig. 1C and D). Notably, KDM3A depletion decreased hypoxia-induced invasion of MCF7 cells by $>2$-fold compared with that of si-CTL cells. This finding implies that KDM3A is involved in hypoxia-induced invasive cell motility. To assess whether the upregulation of KDM3A expression in hypoxia is sufficient to induce cancer cell invasion, and whether other hypoxia-induced signalling pathways are necessary, the effect of KDM3A overexpression on MCF7 cell invasion in normoxic conditions was examined (Fig. 1E and F). The invasive capacity of cells transfected with ectopic Flag-KDM3A increased by $~ 15$-fold compared with that of Mock-transfected cells, even in normoxia. This finding supports the notion that the enhanced expression 
A

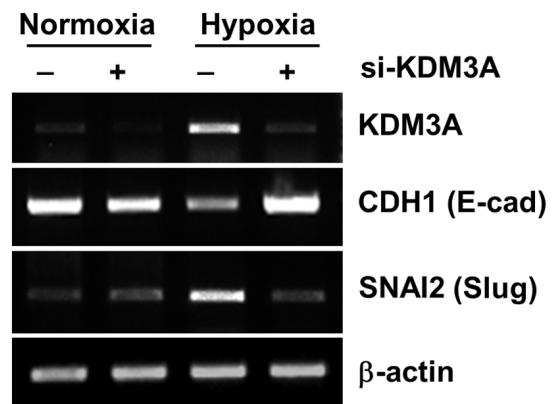

C

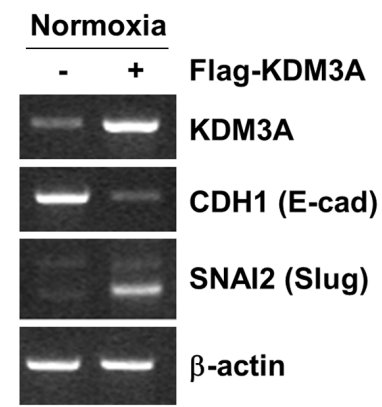

E

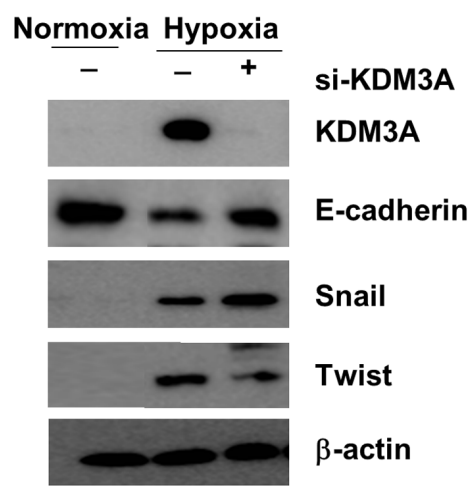

B

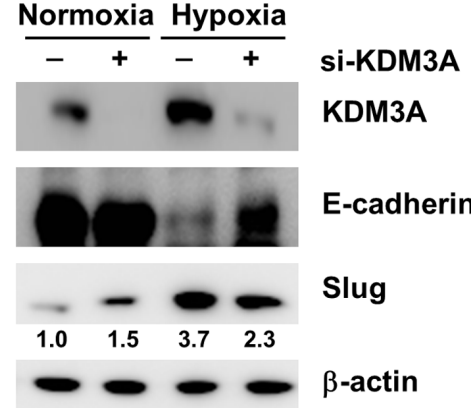

D

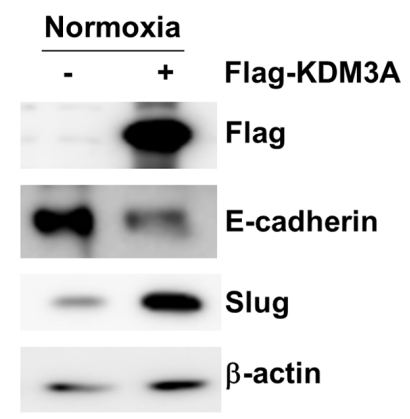

F

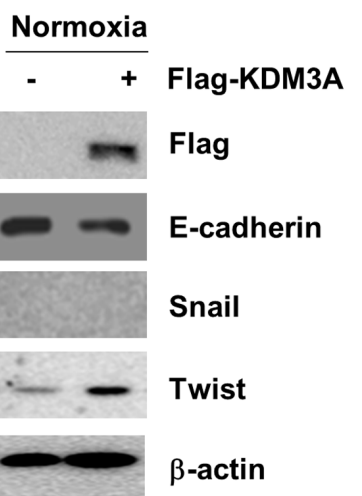

Figure 2. Elevated KDM3A expression in hypoxia upregulates Slug, which suppresses CDH1 expression. (A and B) KDM3A knockdown impairs CDH1 (E-cadherin) downregulation as well as SNAI2 (Slug) upregulation in hypoxia. MCF7 cells transfected with either si-CTL (-) or si-KDM3A (+) were cultured for $48 \mathrm{~h}$ in normoxia. Next, the cells were divided for culture under normoxic $\left(21 \% \mathrm{O}_{2}\right)$ or hypoxic conditions $\left(1 \% \mathrm{O}_{2}\right)$ for $24 \mathrm{~h}$. The expression of mRNA specific to each target gene, as determined by RT-PCR, is displayed in panel A, while the protein expression levels measured by IB using specific antibodies against the proteins indicated in the figure are shown in panel B. (C and D) KDM3A overexpression decreases CDH1 expression while increasing SNAI2 expression in normoxia. MCF7 cells transfected with either empty vector (-) or Flag-tagged KDM3A-encoding vector (+) were cultured for $48 \mathrm{~h}$, and CDH1 and SNAI2 expression at the (C) mRNA and (D) protein level was examined using RT-PCR and IB, respectively. (E) KDM3A knockdown in MCF7 cells decreases Twist upregulation in hypoxia while increasing the hypoxic induction of Snail expression. (F) KDM3A overexpression increases Twist expression in MCF7 cells cultured under normoxic conditions. The experiments were performed as described in (D). si, small interfering; RT-PCR, reverse transcription-PCR; KDM, lysine demethylase; IB, immunoblotting.

of endogenous KDM3A in hypoxic conditions is sufficient to promote hypoxia-induced breast cancer cell invasion. It is notable that ectopic expression of KDM3A had no effect on MCF7 cell invasion in hypoxia. It can be speculated that the robust increase in endogenous KDM3A expression in hypoxia (Fig. 2) may be sufficient to induce cell invasion to the level that is not further enhanced by additional exogenous KDM3A.

KDM3A promotes hypoxia-induced Slug expression. Transcriptional alteration is critical for cancer cells to switch phenotypic states in response to microenvironmental conditions. It was hypothesised that KDM3A-mediated chromatin modification is involved in regulating the transcriptional expression of key players promoting cancer cell invasion. E-cadherin, which is encoded by CDH1, is a well-known epithelial cell marker. CDH1 downregulation is a characteristic of EMT, which induces cell motility (13). Under hypoxic conditions $\left(1 \% \mathrm{O}_{2}\right.$ for $\left.24 \mathrm{~h}\right), \mathrm{CDH} 1$ mRNA expression in MCF7 cells was largely decreased compared with that in cells cultured under normoxic conditions (Fig. 2A). Compromised $\mathrm{CDH} 1$ expression in hypoxic conditions was also observed at the protein level (Fig. 2B). By contrast, KDM3A depletion restored $\mathrm{CDH} 1$ expression under hypoxic conditions to levels comparable to those observed in control cells under normoxic conditions (Fig. 2A and B). This finding implies that KDM3A is necessary for hypoxia-induced downregulation of E-cadherin, which is associated with the induction of cell invasion. 
A
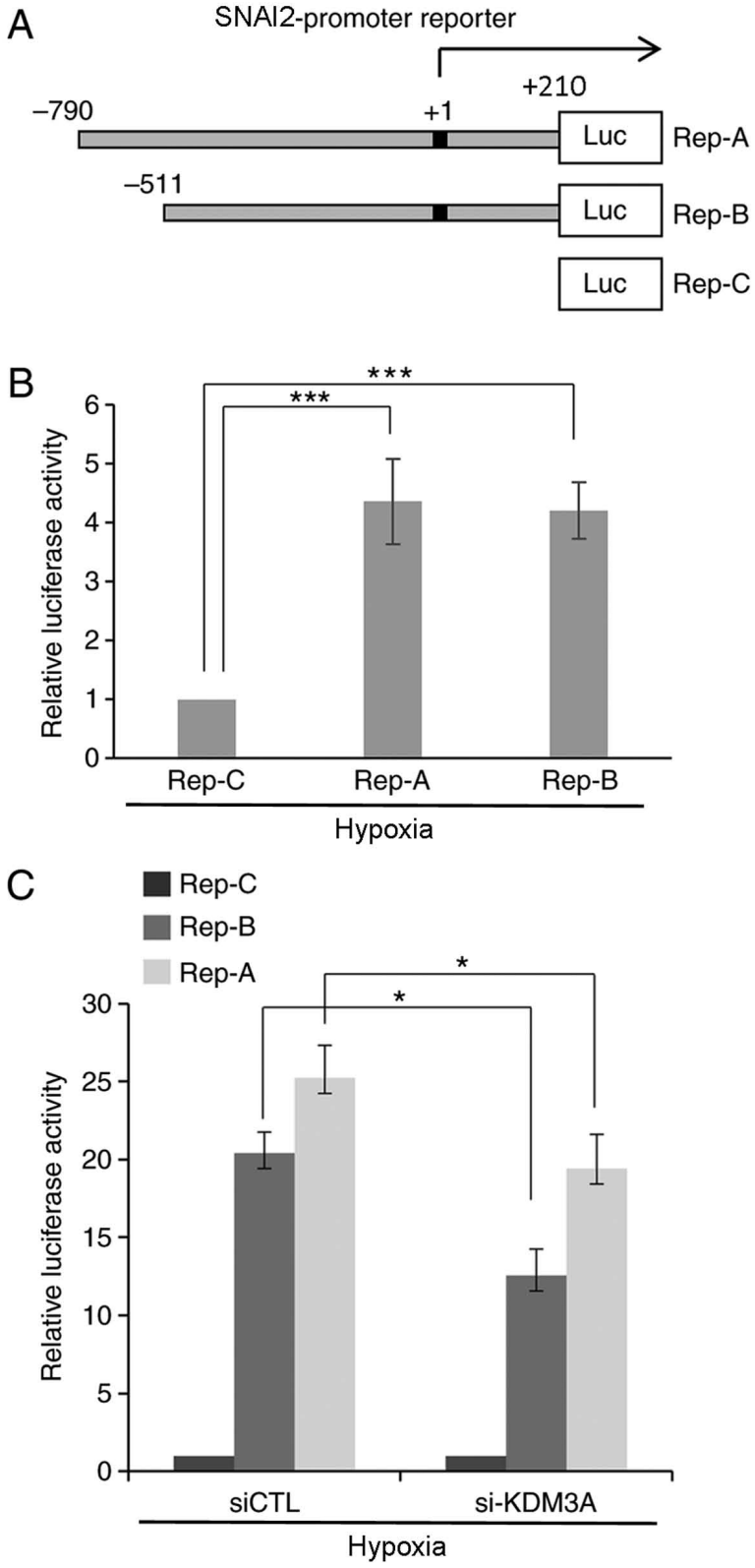

Figure 3. KDM3A regulates SNAI2 promoter activity. (A) Schematic reporter constructs to measure SNAI2 promoter activity. Rep-A encodes a DNA fragment encompassing -790 to +210 bp from the SNAI2 gene transcription start site $(+1)$, which is fused to the coding sequence of the Luc gene. Rep-B lacks a DNA fragment spanning the region -790 to -511 bp from Rep-A. Rep-C only carries Luc. (B) Cells expressing Rep-A and Rep-B displayed prominent promoter activities in hypoxia. MCF7 cells transfected with each reporter construct were cultured for $48 \mathrm{~h}$ in normoxia $\left(21 \% \mathrm{O}_{2}\right)$, followed by an additional $24 \mathrm{~h}$ of culture in hypoxia $\left(1 \% \mathrm{O}_{2}\right)$. Reporter activities were then determined by measuring the luminescence signal generated from Luc. The graph shows an mean of three experiment $\left({ }^{* * * *} \mathrm{P}<0.001\right)$. (C) KDM3A knockdown decreases SNAI2 promoter activity in hypoxia. MCF7 cells transfected with either si-CTL or si-KDM3A were cultured for $48 \mathrm{~h}$ under normoxic conditions. Next, the cells were cultured in hypoxia $\left(1 \% \mathrm{O}_{2}\right)$ for an additional $24 \mathrm{~h}$. The graph displays the mean of three experiments that measured reporter activities with luminescence signal detection. ${ }^{*} \mathrm{P}<0.05$. Luc, luciferase; so, small interfering; KDM, lysine demethylase; CTL, control.

Given that KDM3A removes $\mathrm{H} 3 \mathrm{~K} 9$ methylation, which represses transcription, KDM3A is expected to function as a transcriptional activator. However, enhanced CDH1 expression in KDM3A-depleted hypoxic cells suggests that KDM3A negatively regulates $\mathrm{CDH} 1$ expression. To explain this discordant observation, it was hypothesised that decreased CDH1 expression in hypoxia may be a consequence of KDM3A-mediated activation of a transcription factor involved in $\mathrm{CDH} 1$ expression. Multiple transcription factors are involved in EMT-associated changes in CDH1 expression (14). Among them, Slug, which directly represses CDH1 expression, is induced in hypoxia (15). The present study assessed whether Slug expression was affected by the loss of KDM3A. Consistent with a previous report (15), hypoxic stress $\left(1 \% \mathrm{O}_{2}\right.$ for $\left.24 \mathrm{~h}\right)$ increased Slug expression in MCF7 cells compared with that of control cells. By contrast, KDM3A knockdown led to a prominent decrease in Slug expression levels under hypoxic conditions at both the mRNA and protein levels (Fig. 2A and B). This result suggests that KDM3A upregulates the transcriptional expression of Slug. Consistent with this finding, ectopic overexpression of KDM3A in MCF7 cells increased the expression of Slug, even under normoxic conditions (Fig. 2C and D). In addition, the expression of CDH1 (E-cadherin) at both the mRNA and protein levels was largely decreased by KDM3A overexpression (Fig. 2C and D). Together, these results support that enhanced KDM3A expression upregulates the transcription of Slug, subsequently downregulating $\mathrm{CDH} 1$ expression, which is associated with EMT-associated cell invasion. Furthermore, KDM3A depletion decreased the hypoxia-induced expression of Twist, another EMT-associated transcription factor that represses E-cadherin expression (Fig. 2E). Consistently, the ectopic overexpression of KDM3A increased Twist expression under normoxic conditions (Fig. 2F). By contrast, KDM3A knockdown increased hypoxia-induced Snail expression (Fig. 2E). In normoxic cells transfected with KDM3A-overexpressing constructs, Snail protein expression was not detected (Fig. 2E and F). It is likely that an increase in KDM3A alone is not sufficient to induce Snail expression in normoxia but requires additional factor(s) activated in hypoxia. It was not determined as to whether KDM3A-knockdown affected Snail expression in normoxia, as Snail expression appeared not to be associated with KDM3A-dependent E-cadherin repression. To summarise, these results imply that KDM3A mediates the upregulation of Twist in addition to Slug, both of which are involved in the repression of E-cadherin expression.

KDM3A positively regulates the transcriptional activity of the SNAI2 promoter. To understand the molecular mechanism by which KDM3A upregulates the transcription of Slug, the effect of KDM3A depletion on the promoter of the SNAI2 gene, which encodes Slug, was examined. First, a reporter system carrying an 1 -kb fragment encompassing $790 \mathrm{bp}$ upstream to $210 \mathrm{bp}$ downstream of the SNAI2 TSS (+1) fused to the luciferase coding region (Rep-A) was constructed (Fig. 3A). To further define the promoter of SNAI2, an additional reporter system lacking the DNA region spanning $-790 \mathrm{bp}$ to $-511 \mathrm{bp}$ from the TSS (Rep-B) was generated. A reporter containing only the luciferase gene without a promoter element was used as a negative control (Rep-C). MCF7 cells transfected with each reporter construct were subjected to luciferase assays to assess SNAI2 promoter activity through the expression of luciferase. Cells transfected with both the Rep-A and Rep-B reporters expressed prominent levels of luciferase under hypoxic conditions $\left(1 \% \mathrm{O}_{2}\right)$, as detected by luminescence (Fig. 3B). This 
finding indicates that the cloned DNA fragments 5' upstream of the SNAI2 TSS function as competent promoter elements under hypoxic conditions. Next, KDM3A-specific siRNA along with reporter constructs were simultaneously introduced into MCF7 cells, and reporter activity was measured under the same hypoxic conditions using a quantitative luminescence assay (Fig. 3C). Compared with their activities in si-CTL-transfected cells, cells transfected with si-KDM3A exhibited a statistically significant decrease in the reporter activities of both Rep-A and Rep-B. This finding demonstrates that KDM3A depletion leads to a defect in the transcriptional activity of the SNAI2 promoter under hypoxic conditions.

Dimethylation of histone $\mathrm{H} 3$ at lysine 9 (H3K9me2) at the SNAI2 promoter is regulated by the recruitment of KDM3A. Next, to determine whether KDM3A functions as a histone demethylase to alter the chromatin structure of the SNAI2 promoter, whether histone methylation at the SNAI2 promoter region was altered by the loss of KDM3A was examined. In total, 10 primer pairs specific to regions within the SNAI2 promoter and the downstream adjacent DNA element (from $-1,500$ to $+1,000)$ separated by a 200 -bp interval were designed (Fig. 4A). First, to determine the recruitment of KDM3A to the SNAI2 promoter region, MCF7 cells cultured under normoxic or hypoxic conditions $\left(1 \% \mathrm{O}_{2}\right)$ for $24 \mathrm{~h}$ were subjected ChIP using an anti-KDM3A-specific antibody, followed by PCR-mediated amplification of the antibody-associated DNA regions. As a non-specific enrichment control, anti-IgG was used as a control antibody. The KDM3A-specific ChIP signals were semi-quantitatively measured by normalization to the IgG ChIP signals. The enrichment of KDM3A was observed in three regions, namely regions $\mathrm{d}, \mathrm{B}$ and $\mathrm{g}$, which are located approximately $-1,100,-350$ and -110 bp away from the TSS (+1), respectively. In region d, KDM3A accumulation was detected under both normoxic and hypoxic conditions (Fig. 4B and C). By contrast, KDM3A enrichment at regions $\mathrm{B}$ and $\mathrm{g}$ was specific to hypoxia. All other regions (A, e, f, h, j, C and j) exhibited minimal KDM3A ChIP signalling; implying that, while KDM3A associates with the SNAI2 promoter region $\sim 1,000$ bp upstream of the TSS regardless of the oxygen concentration, additional KDM3A is recruited specifically to the SNAI2 promoter region spanning $\sim 350$ bp to $110 \mathrm{bp}$ upstream of the TSS (encompassing region $\mathrm{B}$ and $\mathrm{g}$ ) under hypoxic conditions.

Next, chromatin modification at the SNAI2 promoter in MCF7 cells was assessed by ChIP against H3K9me2. In hypoxia, $\mathrm{H} 3 \mathrm{~K} 9 \mathrm{me} 2$ levels at region $\mathrm{B}$, where hypoxia-induced KDM3A accumulation was detected (Fig. 4B and C), were largely decreased compared with the levels in the other KDM3A-accumulated regions (region $\mathrm{A}$ and $\mathrm{C}$; Fig. 4D and E). By contrast, when KDM3A was depleted with siRNA, H3K9me2 levels at region B were not decreased in hypoxic conditions compared with that in normoxic conditions. Therefore, $\mathrm{H} 3 \mathrm{~K} 9 \mathrm{me} 2$ at region $\mathrm{B}$ of the SNAI2 promoter is likely to be demethylated by KDM3A, which is specifically recruited under hypoxic conditions (Fig. 4B and C). Thus, this result suggests $\mathrm{H} 3 \mathrm{~K} 9 \mathrm{me} 2-$ mediated transcriptional repression of SNAI2 is relieved under low-oxygen conditions. KDM3A depletion mediated a decrease in $\mathrm{H} 3 \mathrm{~K} 9 \mathrm{me} 2$ at region $\mathrm{B}$ under normoxic conditions (Fig. 4D and E). It is possible that the functional involvement of KDM3A in demethylating the SNAI2 promoter may be distinctive between normoxic and hypoxic conditions, which was not further characterized in the present study.

\section{Discussion}

The phenotypic change of cancer cells in the hypoxic tumour microenvironment promotes aggressive cancer progression (1). Understanding the molecular mechanisms underlying hypoxia-mediated cancer cell state transition will pave the way to develop treatment strategies. Epigenetic modifications to mediate transcriptional alteration are crucial for cellular state transitions (16). Accordingly, attempts have been made to characterize the functional implication of epigenetic modifications, including lysine demethylase-mediated chromatin alterations in hypoxia-mediated transcriptional modulation (17). In this context, the present study demonstrated the functional involvement of KDM3A in the hypoxia-induced metastatic potential of breast cancer cells through increased invasion. Mechanistically, KDM3A, the expression of which is increased by HIF-1 under hypoxic conditions, is recruited to remove the transcriptionally repressive histone modification marker H3K9me2 from the promoter of SNAI2, which encodes Slug, an EMT transcription factor. Consequently, the increased expression of Slug decreases the expression of CDH1-encoded E-cadherin, which is a distinctive characteristic of EMT that confers cancer cell metastatic potential. These results collectively suggest that KDM3A is a transcriptional coactivator of Slug expression that induces cancer cell invasion in hypoxia. In addition, KDM3A is involved in the induction of Twist expression in hypoxia, which also promotes invasive cancer cell motility. Of note, the changes in Slug or Twist expression in the cells with or without KDM3A knockdown were modest compared with those in E-cadherin expression. It is likely that the dysregulation of E-cadherin expression in cells with KDM3A depletion is cooperatively mediated by a simultaneous decrease in Slug and Twist expression following KDM3A knockdown. Future studies may reveal whether KDM3A would be also recruited to the Twist promoter in hypoxia to regulate its chromatin structure.

Consistent with the results of the present study, the transcriptional coactivator activity of KDM3A has previously been observed in rat trophoblast stem cell differentiation under hypoxic conditions by Chakraborty et al (18). By systemic analyses of the upregulated genes in rats exposed to low oxygen, the study found that matrix metallopeptidase 12 (MMP12) is a crucial gene associated with hypoxia-induced cell invasion. In addition, the study showed that, in hypoxia, KDM3A-mediated $\mathrm{H} 3 \mathrm{~K} 9$ demethylation is required for the expression of MMP12, which is not a direct target of HIF-1 (18). This finding implies that KDM3A, the expression of which is upregulated by HIF-1, functions as a transcriptional coactivator to facilitate the expression of genes involved in hypoxia-induced cellular state transitions such as MMP12. Additionally, in urinary bladder cancer, KDM3A cooperates with HIF-1 to enhance glycolysis and thereby promote cancer cell proliferation (19). By demonstrating that the catalytically inactive KDM3A mutant failed to act as a transcriptional coactivator of HIF-1, the bladder cancer study suggests the importance of KDM3A-mediated 
A

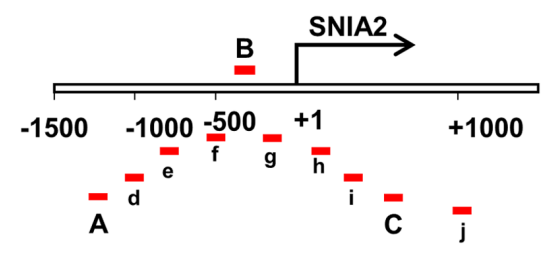

C

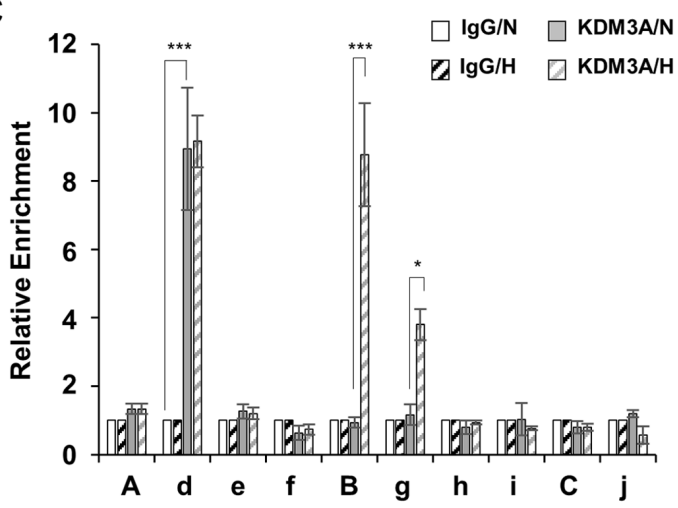

D

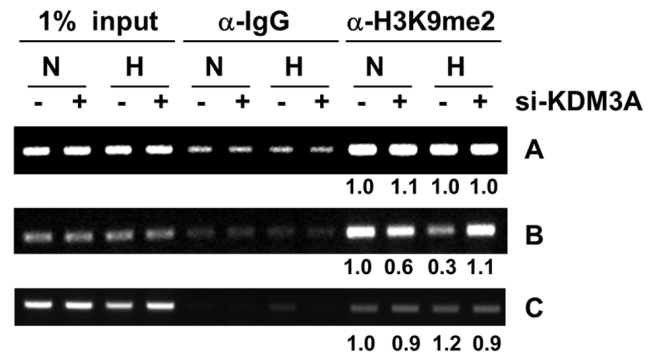

B
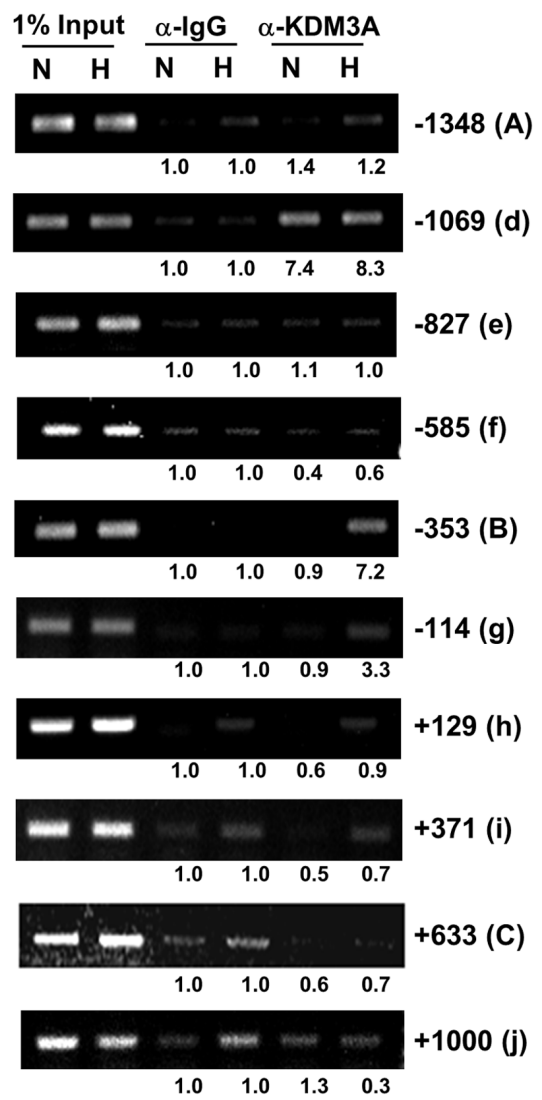

$\mathbf{E}$

\section{$\square$ si-CTL/N $\square$ si-CTL/H \\ $\square$ Si-KDM3A/N $\square$ si-KDM3A/H}

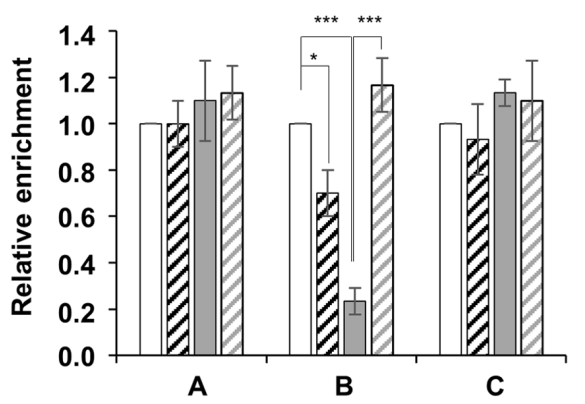

Figure 4. KDM3A recruited to the SNAI2 promoter in hypoxia demethylates H3K9me2 to enhance SNAI2 expression. (A) Schematic drawing of the target regions (A-j) used to assess KDM3A recruitment and H3K9me2 enrichment at the SNAI2 promoter. (B and C) The recruitment of KDM3A to the region $-353 \mathrm{bp}$ (B) from the SNAI2 promoter in MCF7 cells is specific to cells cultured in hypoxic conditions. KDM3A accumulation at chromatin in cells cultured in either normoxia $\left(21 \% \mathrm{O}_{2}\right)$ or hypoxia $\left(1 \% \mathrm{O}_{2}\right)$ for $24 \mathrm{~h}$ was determined by ChIP using an antibody specific to KDM3A or an IgG control antibody. DNA regions associated with KDM3A were assessed by PCR using specific primer pairs to amplify the regions A-j. Input DNA (1\%) served as the control. KDM3A enrichment was semi-quantitatively measured by ImageJ. (B) Anti-KDM3A ChIP-PCR signals were normalized to the anti-IgG ChIP signals from the same regions. The representative image of three different experiments is shown in panel $\mathrm{B}$. The quantitative results reflecting the mean of three experiments are plotted as a bar graph in panel C. " $\mathrm{P}<0.05$ and ${ }^{* * *} \mathrm{P}<0.001$. (D and E) H3K9me2 levels are decreased at the SNAI2 promoter in KDM3A-depleted MCF7 cells cultured under hypoxic conditions. Cells transfected with either si-KDM3A (+) or si-CTL (-) were cultured for $48 \mathrm{~h}$ under normoxic conditions, followed by an additional $24-\mathrm{h}$ culture under normoxic or hypoxic $\left(1 \% \mathrm{O}_{2}\right)$ conditions. $\mathrm{H} 3 \mathrm{~K} 9 \mathrm{me} 2$ enrichment was determined by ChIP-PCR using an antibody specific to H3K9me2, as described in panels B and C. The representative image of three different experiments is shown in panel D and the quantitative results reflecting the mean of three experiments are plotted as a bar graph in panel $\mathrm{E}$. The quantitative results of the anti-H3K9me2 ChIP signals are plotted as a bar graph in panel $\mathrm{E}$. ${ }^{*} \mathrm{P}<0.05$ and ${ }^{* * * *} \mathrm{P}<0.001$. H3K9me2, dimethylation of histone H3 at lysine 9; ChIP, chromatin immunoprecipitation; KDM, lysine demethylase; CTL, control; N, normoxia; H, hypoxia.

chromatin modification in the transcriptional regulation associated with aggressive cancer progression in the hypoxic tumour microenvironment. The KDM3A-mediated decrease in $\mathrm{H} 3 \mathrm{~K} 9 \mathrm{me} 2$ at the SNAI2 promoter in hypoxic breast cancer cells observed in the present study also strongly supports the involvement of KDM3A-dependent chromatin modifications in hypoxic cancer progression.
The involvement of KDM3A in aggressive cancer cell phenotypes was also reported in an aggressive form of breast cancer in a previous study (20). In contrast to the results of the present study demonstrating the necessity of KDM3A in the induction of cellular invasion under hypoxic conditions, the aforementioned study showed that, in the metastatic breast cancer cell lines MDA-MB-231 and Hs578T, KDM3A 
knockdown inhibited cell invasion under normoxic conditions by demethylating $\mathrm{H} 3 \mathrm{~K} 9$ at pro-invasive genes such as MMP9 and S100A4. This result, along with our finding that KDM3A is required for the hypoxic induction of breast cancer cell invasion likely through promoting the expression of Slug, an EMT-associated transcription factor, suggests that KDM3A not only establishes but also maintains the metastatic potential of breast cancer cells. Notably, while Wade et al (21) reported the requirement of KDM3A for estrogen receptor signalling-dependent proliferation of MCF7 breast cancer cells in oestradiol-supplemented medium, the present study failed to observe the detrimental effect of KDM3A on MCF7 cell survival or proliferation in high-glucose DMEM (Fig. 1A and B). It can be speculated that the requirement of KDM3A for MCF7 breast cancer cell survival and proliferation may be largely dependent on growth conditions The mechanism by which KDM3A is recruited to distinctive target genes in different oxygen concentrations such as MDA-MB-231 in normoxia vs. MCF7 in hypoxia, remains unknown. Future studies to identify KDM3A-interacting transcription factors that promote cancer cell invasion will reveal detailed information about hypoxia-induced transcriptional alterations associated with the transition of cancer cells to a more aggressive state.

\section{Acknowledgements}

The authors would like to thank Dr Hyun-Soo Cho (Korea Research Institute of Bioscience and Biotechnology, Daejeon, Republic of Korea) for providing the Flag-KDM3A construct.

\section{Funding}

The present study was supported by the National Research Foundation of Korea funded by the Ministry of Science and Information \& Communication Technology (grant nos. NRF-2013R1A1A1006638 and NRF-2019R1A2C1086151) and by the KRIBB Research Initiative Program.

\section{Authors' contributions}

HJA and JAK conceived and designed the study. HJA, BM, and MP performed the experiments and analyzed the data. JAK wrote the manuscript. All authors read and approved the final manuscript and agreed to be accountable for all aspects of the research.

\section{Availability of data and materials}

The datasets used and/or analysed during the current study are available from the corresponding author on reasonable request.

\section{Ethics approval and consent to participate}

Not applicable.

\section{Patient consent for publication}

Not applicable.

\section{Competing interests}

The authors declare that they have no competing interests.

\section{References}

1. Rankin EB, Nam JM and Giaccia AJ: Hypoxia: Signaling the Metastatic Cascade. Trends Cancer 2: 295-304, 2016.

2. Eales KL, Hollinshead KE and Tennant DA: Hypoxia and metabolic adaptation of cancer cells. Oncogenesis 5: e190, 2016.

3. Chouaib S, Messai Y, Couve S, Escudier B, Hasmim M and Noman MZ: Hypoxia promotes tumor growth in linking angiogenesis to immune escape. Front Immunol 3: 21, 2012.

4. Semenza GL: Hypoxia-inducible factors in physiology and medicine. Cell 148: 399-408, 2012.

5. Xia X, Lemieux ME, Li W, Carroll JS, Brown M, Liu XS and Kung AL: Integrative analysis of HIF binding and transactivation reveals its role in maintaining histone methylation homeostasis. Proc Natl Acad Sci USA 106: 4260-4265, 2009.

6. Hancock RL, Dunne K, Walport LJ, Flashman E and Kawamura A: Epigenetic regulation by histone demethylases in hypoxia. Epigenomics 7: 791-811, 2015.

7. Luo W, Chang R, Zhong J, Pandey A and Semenza GL: Histone demethylase JMJD2C is a coactivator for hypoxia-inducible factor 1 that is required for breast cancer progression. Proc Natl Acad Sci USA 109: E3367-E3376, 2012.

8. Mimura I, Nangaku M, Kanki Y, Tsutsumi S, Inoue T, Kohro T, Yamamoto S, Fujita T, Shimamura T, Suehiro J, et al: Dynamic change of chromatin conformation in response to hypoxia enhances the expression of GLUT3 (SLC2A3) by cooperative interaction of hypoxia-inducible factor 1 and KDM3A. Mol Cell Biol 32: 3018-3032, 2012.

9. Lee HY, Yang EG and Park H: Hypoxia enhances the expression of prostate-specific antigen by modifying the quantity and catalytic activity of Jumonji C domain-containing histone demethylases. Carcinogenesis 34: 2706-2715, 2013.

10. Park SJ,Kim JG, Son TG, Yi JM, Kim ND, Yang K and Heo K: The histone demethylase JMJD1A regulates adrenomedullin-mediated cell proliferation in hepatocellular carcinoma under hypoxia. Biochem Biophys Res Commun 434: 722-727, 2013.

11. Ueda J, Ho JC, Lee KL, Kitajima S, Yang H, Sun W, Fukuhara N, Zaiden N, Chan SL, Tachibana M, et al: The hypoxia-inducible epigenetic regulators Jmjd1a and G9a provide a mechanistic link between angiogenesis and tumor growth. Mol Cell Biol 34: 3702-3720, 2014

12. Favaro E, Lord S, Harris AL and Buffa FM: Gene expression and hypoxia in breast cancer. Genome Med 3: 55, 2011.

13. Lamouille S, Xu J and Derynck R: Molecular mechanisms of epithelial-mesenchymal transition. Nat Rev Mol Cell Biol 15: 178-196, 2014.

14. Serrano-Gomez SJ, Maziveyi M and Alahari SK: Regulation of epithelial-mesenchymal transition through epigenetic and post-translational modifications. Mol Cancer 15: 18, 2016.

15. Jiang J, Tang YL and Liang XH: EMT: A new vision of hypoxia promoting cancer progression. Cancer Biol Ther 11: 714-723, 2011.

16. Flavahan WA, Gaskell E and Bernstein BE: Epigenetic plasticity and the hallmarks of cancer. Science 357: eaal2380, 2017.

17. Perez-Perri JI, Acevedo JM and Wappner P: Epigenetics: New questions on the response to hypoxia. Int J Mol Sci 12: 4705-4721, 2011.

18. Chakraborty D, Cui W, Rosario GX, Scott RL, Dhakal P, Renaud SJ, Tachibana M, Rumi MA, Mason CW, Krieg AJ, et al: HIF-KDM3A-MMP12 regulatory circuit ensures trophoblast plasticity and placental adaptations to hypoxia. Proc Natl Acad Sci USA 113: E7212-E7221, 2016.

19. Wan W, Peng K, Li M, Qin L, Tong Z, Yan J, Shen B and Yu C: Histone demethylase JMJD1A promotes urinary bladder cancer progression by enhancing glycolysis through coactivation of hypoxia inducible factor $1 \alpha$. Oncogene 36: 3868-3877, 2017.

20. Ramadoss S, Guo G and Wang CY: Lysine demethylase KDM3A regulates breast cancer cell invasion and apoptosis by targeting histone and the non-histone protein p53. Oncogene 36: 47-59, 2017.

21. Wade MA, Jones D, Wilson L, Stockley J, Coffey K, Robson CN and Gaughan L: The histone demethylase enzyme KDM3A is a key estrogen receptor regulator in breast cancer. Nucleic Acids Res 43: 196-207, 2015. 